\title{
HIGH RESOLUTION SOFT X-RAY BENDING MAGNET BEAMLINE 9.3.2 WITH CIRCULARLY POLARIZED RADIATION CAPABILITY AT THE ADVANCED LIGHT SOURCE*
}

\author{
Z. Hussaina, W.R.A Huffa,b, S.A. Kellara,b, E.J. Molera, b, P.A. Heimann", \\ W.R. McKinney a, H.A. Padmorea, C.S. Fadleya,c, D.A. Shirleyd \\ a Advanced Light Source \\ Lawrence Berkeley National Laboratory \\ University of California \\ Berkeley, CA 94720 \\ b'The University of California \\ Department of Chemistry \\ Berkeley, CA 94720 \\ cThe University of California \\ Department of Physics \\ Davis, CA 95616 \\ dThe Pennsylvania State University \\ Department of Chemistry and Physics \\ University Park, PA 16802
}

Paper submitted at the 11th International Conference on Vacuum Ultraviolet

Radiation Physics, Tokyo, Japan, August 27 - September 1, 1995

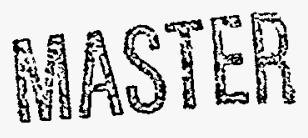

*This work was supported by the Director, Office of Energy Research, Office of Bàsic Energy Sciences, Materials Sciences Division, of the U.S. Department of Energy, under Contract No. DE-AC03-76SF00098. 


\section{DISCLAIMER}

This report was prepared as an account of work sponsored by an agency of the United States Government. Neither the United States Government nor any agency thereof, nor any of their employees, make any warranty, express or implied, or assumes any legal liability or responsibility for the accuracy, completeness, or usefulness of any information, apparatus, product, or process disclosed, or represents that its use would not infringe privately owned rights. Reference herein to any specific commercial product, process, or service by trade name, trademark, manufacturer, or otherwise does not necessarily constitute or imply its endorsement, recommendation, or favoring by the United States Government or any agency thereof. The views and opinions of authors expressed herein do not necessarily state or reflect those of the United States Government or any agency thereof. 

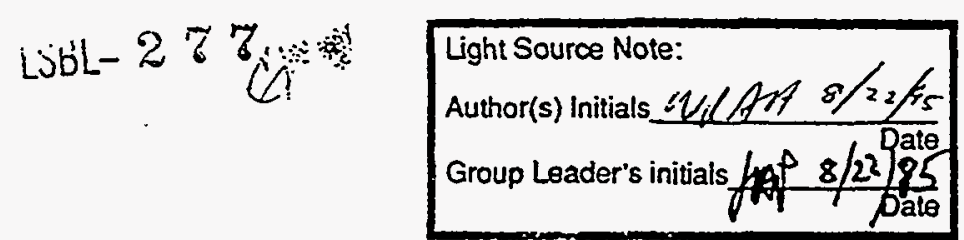

\title{
High Resolution Soft X-Ray Bending Magnet Beamline 9.3.2 with Circularly Polarized Radiation Capability at the Advanced Light Source
}

\author{
Z. Hussain, ${ }^{a}$ W.R.A. Huff, ${ }^{a, b}$ S.A. Kellar, ${ }^{2, b}$ E.J. Moler, ${ }^{a b}$ P.A. Heimann, ${ }^{a}$ W. McKinney, ${ }^{a}$ \\ H.A. Padmore, ${ }^{2}$ C.S. Fadley, ${ }^{a, c}$ D.A. Shirley ${ }^{d}$ \\ aLawrence Berkeley National Laboratory, Berkeley, CA 94720 \\ 'The University of Califomia, Dept. of Chemistry, Berkeley, CA 94720 \\ The University of Califomia, Dept. of Physics, Davis, CA 95616 \\ dThe Pennsylvania State University, Dept. of Chemistry and Physics, University Park, PA 16802
}

Bending magnet beamline 9.3.2 at the Advanced Light Source (ALS) was designed for high resolution spectroscopy in the soft $x$-ray energy region, covering a range from $30 \mathrm{eV}$ to $1500 \mathrm{eV}$ with three gratings. The monochromator itself is a standard fixed included angle $55 \mathrm{~m}$ spherical grating monochromator and was originally used at the Stanford Synchrotron Radiation Laboratory (SSRL) as a prototype for later insertion device based monochromators for the ALS. For operations at the ALS, the toroidal pre-mirror used at SSRL to vertically focus onto the entrance slit and horizontally focus onto the exit slit was replaced by two separate crossed mirrors (Kirkpatrick-Baez configuration).

Circularly polarized radiation is obtained by inserting a water-cooled movable aperture in front of the vertically focusing mirror to allow selecting the beam either above or below the horizontal plane. To maintain a stable beam intensity. through the entrance slit, the photocurrent signals from the upper and lower jaws of the entrance slit are utilized to set a feedback loop with the vertically deflecting mirror Piezoelectric drive. The beamline end station has a rotatable platform (through $60^{\circ}$ ) that accommodates two experimental chambers, enabling the synchrotron radiation to be directed to either one without breaking vacuum.

\section{INTRODUCTION}

BL 9.3.2 is a Rowland circle SGM installed on a bending magnet and its energies extend from 30 $\mathrm{eV}$ to $1500 \mathrm{eV}$. Originally BL 6-1 on a 55-pole wiggler at SSRL, BL 9.3.2 was developed as a prototype for insertion device monochromators at the ALS. ${ }^{1}$ Heimann et al. ${ }^{2}$ describe the beamline design and performance while installed at SSRL.

Besides adopting the Kirkpatrick-Baez (KB) configuration, ${ }^{3,4}$ several other improvements were made to $B L$ 9.3.2. Bending magnet radiation offers the option of choosing linearly as well as circularly or elliptically polarized light. An aperture to select part of the beam above or below the plane of the storage ring allows the polarization selection. Additionally, photon beam instabilities are corrected in real time by an active feedback loop connected to the vertically deflecting mirror. This beam position locking has greatly improved the photon beam stability at the end station. Finally, because the end stations must maintain UHV, a double-chamber rotatable platform was installed so that they would not have to be vented to go on-line.

\section{OPTCS}

Fig. 1 is a schematic of the beamline which has a base pressure better than $60 \mathrm{nPa}$. The vacuum chambers are supported by the AlS orthogonal six- strut sytem designed to withstand $1 \mathrm{~g}$ of lateral acceleration. The stands for the mirrors and slits have a first vibrational mode higher than $30 \mathrm{~Hz}$. The grating tank stands are filled with water for thermal stability.

The optics were surveyed to within $100 \mu \mathrm{m}$ of the desired position based on ray tracing analyses of the beamline. Further alignment was completed by measuring the $N_{2(\mathrm{~g})} 1 s$ to $\pi^{*}$ resonance to monitor the resolution as well as scanning the intensity at various points along the beamline.

Kirkpatrick-Baez Deflection Mirrors: $\mathrm{M} 1$ is a water cooled tangential cylinder accepting 7.5 mrads of the horizontal radiation fan; this is determined by the length $(1.2 \mathrm{~m})$ and radiation incidence angle $\left(2.5^{\circ}\right)$. M1 focuses the beam horizontally near the exit slit (S2) with a magnification of 2.5. The M1 radius was designed to be $242 \mathrm{~m}$; the delivered radius was $234 \mathrm{~m}$. The focus was thus moved closer to the mirror by $2.7 \mathrm{~m}$.

Completing the $\mathrm{KB}$ design is the sperical mirror M2 (242 m radius) which focuses the beam vertically at the midpoint of the entrance slit (S1) travel with a magnification of 0.60 . M2 accepts 1.2 mrads of the vertical radiation fan and the angle of incidence is $2.5^{\circ}$. The vertical beam should be focused at S1 to obtain high throughput with a narrow slit width for maximum resolution. The M2 focal point was measured using a photodiode 


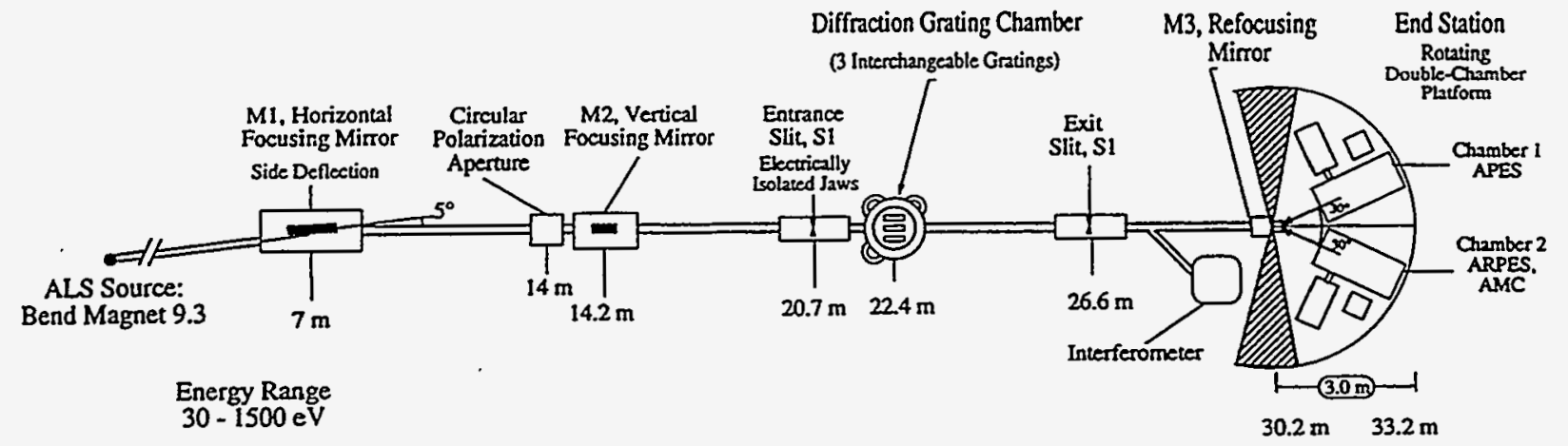

Figure 1: ALS Beamline 9.3.2 Schematic

directly downstream of S1. With S1 at $10 \mu \mathrm{m}$, the M2 pitch was adjusted to scan the photon beam profile across the slit gap. The SI position was changed after each scan and thus the focal point was experimentally determined to be $8.52 \mathrm{~m}$ from $\mathrm{M} 2$ ( $0.10 \mathrm{~m}$ toward the grating tank from the design specification).

Gratings: Using one of three gratings, the accessible energy range is $30 \mathrm{eV}$ to $1500 \mathrm{eV}$. The gratings are kinematically mounted onto a carriage attached to a rail by ball bearing rollers. ${ }^{1}$ Fig. 2 plots the flux vs. energy for each grating as measured from a gold photodiode downstream from S2. Each spherical grating is designed to have a 55 $\mathrm{m}$ radius. The fixed inclusion angle is $174^{\circ}$. The grating angle position is monitored by a laser interferometer. ${ }^{1}$ The fact that the flux from the high energy grating does not drop off may indicate a large scattered light component.

The Refocussing Mirror, M3: The present M3 is a bent cylinder with an adjustable large radius. This is being replaced by a toroid having a fixed small radius $(6.0 \mathrm{~cm})$ and an adjustable large radius (100$150 \mathrm{~m}$ ). The bending mechanism is based on a design by Howells ${ }^{5}$ and will allow for moving the focus from $1.5 \mathrm{~m}$ to $2.5 \mathrm{~m}$ from $\mathrm{M} 3$.

The following table summerizes the optical qualities of $M 1, M 2$, and $M 3$ :

\begin{tabular}{|c|c|c|}
\hline Mirror & RMS Slope Enor & Surface Roughness \\
\hline M1 & $<10 \mu \mathrm{rads}$ & $-5 \AA$ \\
M2 & $<0.6 \mu \mathrm{rads}$ & $-3 \AA$ \\
M3 & $<10$ prads & $-5 \AA$ \\
\hline
\end{tabular}

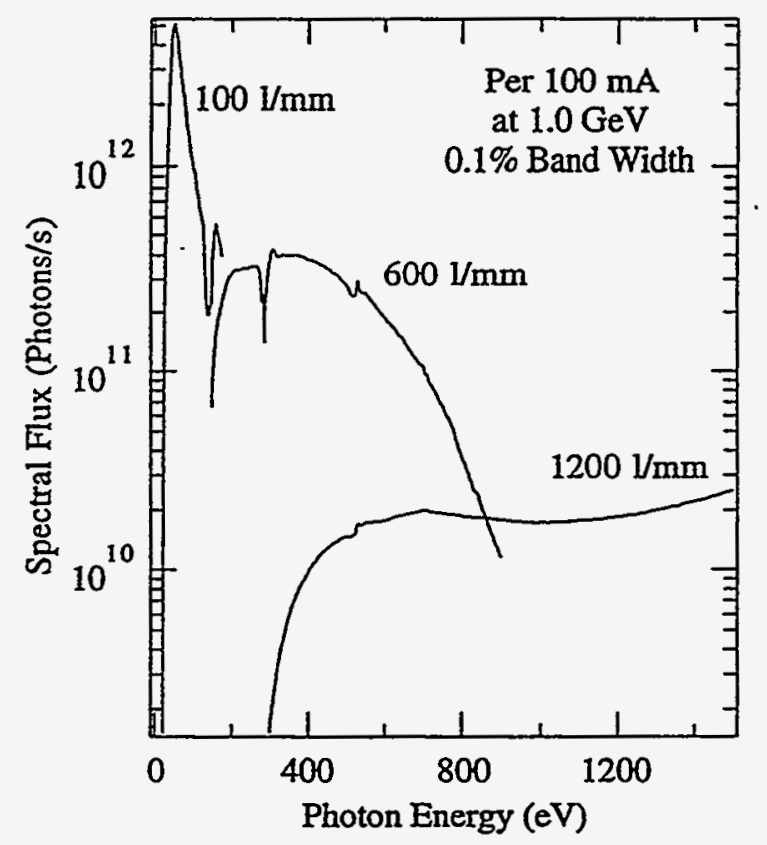

Fig. 2. Flux vs. Photon Energy

\section{SLITS}

Each slit is based on a flexure design allowing a side-driven micrometer to push the jaws open against spring tension continuously from $\leq 3$ $\mu \mathrm{m}$ to $1500 \mu \mathrm{m}$ maintaining the jaws parallel. The jaws must also be parallel with the grating lines or else the slit width is effectively widened and the energy resolution is degraded. 
To satisfy the Rowland circle condition over a wide energy range, the entrance and exit slits are translatable over $600 \mathrm{~mm}$ and $1000 \mathrm{~mm}$, respectively. BL 9.3.2 has three modes of operation: fixed slits, scanned slits (Rowland circle), and scanned exit slit (focus condition). The fixed slits mode is appropriate when a small energy range is being scanned. For best resolution, the slits should be set as close to the Rowland circle or a focus condition as possible. The following table gives the energy range for each grating under the Rowland circle condition.

Energy Range in Rowland Circle Mode

\begin{tabular}{|ccc|}
\hline Grating & $\mathrm{E}_{\min .}(\mathrm{eV})$ & $\mathrm{E}_{\max .}(\mathrm{eV})$ \\
\hline 100 & 42.1 & 69.2 \\
600 & 252.8 & 415.3 \\
1200 & 505.6 & 830.7 \\
\hline
\end{tabular}

These ranges are limited by the entrance slit's translation limits. The full energy range of each grating can be reached under the focus condition by moving S1 away from the M2 focus and thus sacraficing some flux.

It is critical that the slits' translation lie along the photon beam path. If S1 does not, then more or less of the beam centroid will be accepted which will adversely affect the flux. If the S2 pitch is wrong, then the $S 2$ position will be incorrect for a given photon energy. Also, if S1 and/or S2 do not travel along the beam path, then the energy calibration will change due to the changing inclusion angle.

Satisfying the focus condition and closing $\mathrm{S} 1$ and $\mathrm{S} 2$ to $10 \mu \mathrm{m}$ each, the resolving power is $E / \Delta E \geq 7,000$. Fig. 3 plots the $\mathrm{N}_{2(\mathrm{~g})}$ 1s to $\pi^{*}$ resonance using first order light from the $600 \mathrm{l} / \mathrm{mm}$ grating. For this spectrum, the slit positions were set to satisfy the grating focus condition and remained fixed during the scan.

\section{BEAMLINE ATTRIBUTES}

Circular Polarization: A water-cooled aperture is installed upstream of $\mathrm{M} 2$ which can be positioned to select the beam centroid for linear polarized light. Alternatively, the aperture can be positioned above or below the beam center to select circularly or eliptically polarized light. The degree of circular polarization as measured at the endstation is over 0.8 at $700 \mathrm{eV}$ with $\geq 30 \%$ of the total flux. ${ }^{6}$

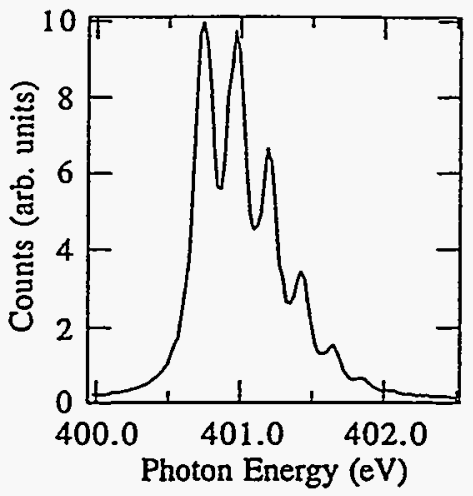

Fig. 3. $N_{2(g)} 1 s$ to $\pi^{*}$

Rotating Platform Endstation: The endstations are mounted on a rotating platform to maximize the efficiency of changing endstations. Permanently mounted at station 1 is the Advanced Photoelectron Spectrometer for doing a variety of surface science experiments. Mounted at station two is usually the Applied Materials Chamber for doing NEXAFS and photoemission measurements. Alternately mounted at station two is the Angle-Resolved Photoemission Spectrometer used for studying surfaces and interfaces.

The two halves of the platform are vibrationally decoupled from one another to allow assembly of one endstation while the other takes beam. The platform rotates manually through $60^{\circ}$ in $<5$ min.; the electronics have been connected appropriately. The rotation stops have been designed to align the chambers upon successive rotations. For structural stability, the chambers are bolted to pods secured to the floor removing the 'drum-head' effect of the large platform.

Photodiodes: A photodiode is installed downstream from each optical component to aid alignment and storage ring diagnostics. These are electrically isolated to allow for photocurrent measurements and/or coated with phosphor for viewing the beam. The parts used to collect photocurrent signals expose a clean gold surface to the beam. Thus, absolute flux calculations can be performed.

I-zero: Directly downstream of M3 are a gold grid and a copper grid ( $>85 \%$ transmission). Evaporators for each metal are maintained so that a fresh layer can be deposited on the respective grid and a clean Izero signal can be collected. These grids are 
electrically isolated and the I-zero photoecurrent can be measured directly. A potential may be applied to an electrically isolated wire loop surrounding the face of the grids to collect all photoelectrons and thus improve the stability and accuracy of the measured photocurrent. Alternatively, one can measure total yield by using a channelton positioned $90^{\circ}$ to the beam.

Active Feedback on M2 Pitch: The upper and lower jaws of SI are electrically isolated from each other and from ground. Thus, the photocurrent from each jaw at a given slit width can be monitored. This signal then goes through a feedback loop (PID logic) which is used to automatically adjust a piezoelectric drive which in turn changes the M2 pitch. The same fraction of the beam makes it through S1 provided the same fraction of the beam is maintained on the upper and lower jaws.

Fig. 4 plots the error function, $E_{f}$, used for the feedback loop as a function of time. $I_{U}$ and $I_{\mathrm{L}}$ indicate the photocurrent on the upper and lower jaws, respectively. Without the feedback loop in operation, the beam can drift causing the changes in the photocurrent collected from each jaw. However, when in operation, the feedback loop effectively locks the beam position thus stabilizing the flux at the endstation.

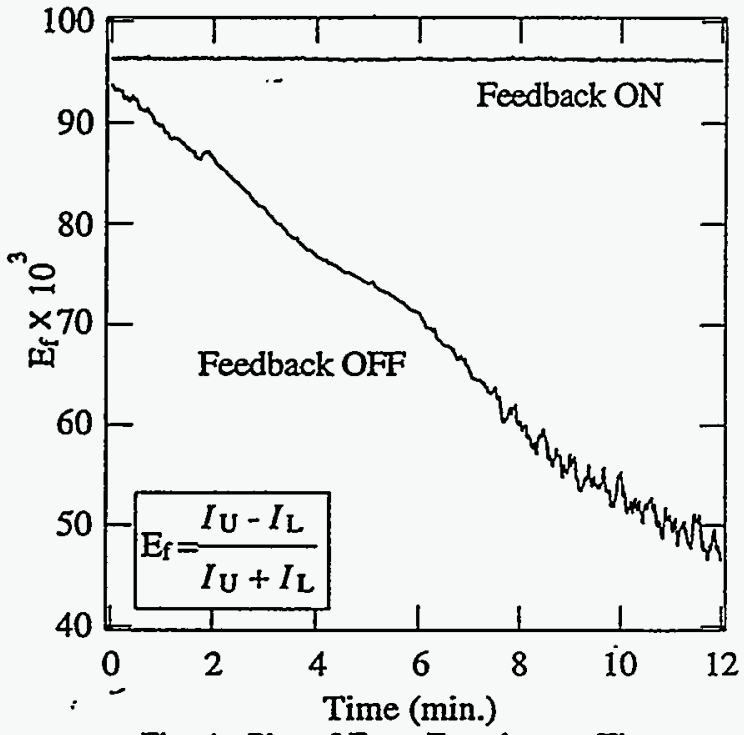

Fig. 4. Plot.of Error Function vs. Time Illustrating Feedback Operation
The purpose for developing this beam position locking was to correct for photon beam fluctuations caused by many factors. These may include instability of the electron beam in the storage ring, dimensional changes in the positions of the optics due to vibration, and temperature variations of the low conductivity water (LCW) used to cool the ALS magnets and some optics. Originally, the LCW temperature varied $\sim \pm 0.5 \mathrm{C}^{\circ}$ which caused large fluctuations in the photon beam-an intensity oscillation $- \pm 1 \%$ with a $\sim 10 \mathrm{~min}$. period. Successful efforts constrained the LCW temperature to within $\pm 0.1 \mathrm{C}^{\circ}$ and the photon beam has become extremely stable $(0.1 \%$ to $0.2 \%)$.

\section{ACKNOWLEDGEMENTS}

We wish to thank the ALS designers, engineers, and technicians. Special thanks must be extended to Ted Lauritzen, Curtis Cummings, and Pat McKean. This work was supported by U.S. DOE contract number DE-AC03-76SF00098.

\section{REFERENCES}

${ }^{1}$ W.R. McKinney, M.R. Howells, T. Lauritzen, J. Chin, R. DiGennaro, E. Fong, W. Gath, J. Guigli. H. Hogrefe, J. Meneghetti, D. Plate, P.A. Heimann, L. Terminello, Z. Ji, D.A. Shirley, and F. Senf, Nuc. Instrum. and Meth. in Phys. Research, A291 (1990)221-224.

${ }^{2}$ P.A. Heimann, F. Senf, W. McKinney, M. Howells, R.D. van Zee, L.J. Medhurst, T. Lauritzen, J. Chin, J. Meneghetti, W. Gath, H. Hogrefe, and D.A. Shirley, Physica Scripta T31 (1990)127-130.

${ }^{3}$ A.G. Michette, Optical Systems for Soft X-Rays, Plenum Press, New York, 1986.

${ }^{4}$ C. Kunz (ed.), Topics in Current Physics: Synchrotron Radiation, Techniques and Applications, Springer-Verlag, Berlin, Heidelberg (1979).

${ }^{5} \mathrm{M}$. Howells, Unpublished.

${ }^{6}$ J.B. Kortright, M.E. Rice Z. Hussain, H.A. Padmore, A. Adamson, W.R.A. Huff, E.J. Moler, S.A. Kellar, R.X. Ynzunza, F.J. Palomares, H. Daimon, E.D. Tober, and C.S. Fadley, To Be Published, SRI 1995, USA. 